P. Montaldo et al.

\title{
Cooling in a low-resource environment: lost in translation
}

Paolo Montaldo ${ }^{\mathrm{a}}$, Shreela S. Pauliah ${ }^{\mathrm{a}}$, Peter J. Lally ${ }^{\mathrm{a}}$, Linus Olson ${ }^{\mathrm{b}}$, Sudhin Thayyil ${ }^{\mathrm{a},{ }^{*}}$

${ }^{a}$ Centre for Perinatal Neuroscience, Imperial College London, Hammersmith Hospital, London, UK

${ }^{\mathrm{b}}$ Department of Women's and Children's Health, Karolinska Institutet, Stockholm, Sweden

${ }^{*}$ Corresponding author. Address: Centre for Perinatal Neuroscience, Level 5, Hammersmith House, Department of Paediatrics, Imperial College London and Imperial College Healthcare NHS Trust, Du Cane Road, London W12 0HS, UK. Tel.: +44 2033138515.

E-mail address: s.thayyil@imperial.ac.uk (S. Thayyil).

\section{SUMMARY}

Although cooling therapy has been the standard of care for neonatal encephalopathy (NE) in highincome countries for more than half a decade, it is still not widely used in low- and middle-income countries (LMIC), which bear 99\% of the encephalopathy burden; neither is it listed as a priority research area. Here we explore the major roadblocks that prevent the use of cooling in LMIC, including differences in population comorbidities, suboptimal intensive care, and the lack of affordable servo-controlled cooling devices. The emerging data from LMIC suggest that the incidence of coexisting perinatal infections in NE is no different to that in high-income countries, and that cooling can be effectively provided without tertiary intensive care and ventilatory support; however, the data on safety and efficacy of cooling are limited. Without adequately powered clinical trials, the creeping and uncertain introduction of cooling therapy in LMIC will be plagued by residual safety concerns, and any therapeutic benefit will be even more difficult to translate into widespread clinical use.

Keywords:

Therapeutic hypothermia

Low- and middle-income countries

Neonatal encephalopathy

Neonatal mortality

\section{Introduction}

Perinatal asphyxia associated with moderate to severe neonatal encephalopathy (NE) occurs at an approximate rate of 1-2 per 1000 live births in high-income countries [1] and 10-20 per 1000 live births in low- and middle-income countries (LMIC) [2]. Following moderate or severe encephalopathy, $\sim 25-60 \%$ of the affected infants die and more than half of the survivors sustain 
significant brain injury and lifelong disability in LMIC [3]. Of the one million annual neonatal deaths caused by perinatal asphyxia, $99 \%$ occur in LMIC [4].

Therapeutic hypothermia has become the standard of care for NE in high-income countries following two decades of rigorous experimental and clinical research. This began with the demonstration of secondary energy failure and its amelioration with therapeutic hypothermia in animal models, followed by clinical trials, meta-analyses, registries, and finally its inclusion in national and international guidelines [5]. Therapeutic hypothermia reduces mortality after NE [typical relative risk (RR): $0.75 ; 95 \%$ confidence interval (CI): $0.64-0.88$ ] and neurodisability in survivors at 18 months (typical RR: 0.77; 95\% CI: 0.63-0.94) [6] and at school age (typical RR: 0.59; 95\% CI: 0.37-0.94) [7,8]. Almost all eligible babies receive therapeutic hypothermia in the UK at present ( $~ 800$ per year), and this is estimated to have saved the National Health Service a total of $£ 125 \mathrm{~m}$ since 2009 [9].

Given the simplicity of the intervention and the global disease burden, therapeutic hypothermia may have a considerable impact on the health and economies in LMIC. Unfortunately, in these settings the uptake of therapeutic hypothermia has been poor. Here we examine the various factors which have prevented the use of this highly effective therapy in settings which shoulder the greatest burden.

\section{Issues related to healthcare infrastructure}

\subsection{Home deliveries and the lack of transport systems}

In the 'standard model' of perinatal services practised in high-income countries, critically ill newborns are rapidly transported to resource-intensive tertiary neonatal units for therapeutic hypothermia - this is not readily applicable to the LMIC. In fact, such models are neither feasible nor desirable in LMIC, where simpler, affordable, and cost-effective interventions in primary and secondary care may be more effective in reducing neonatal mortality. In low-income countries in regions such as Sub-Saharan Africa, some communities have no access by road; communication systems are weak; many cannot afford private transport [10]; and many deliveries happen at home or in poorly equipped facilities [11]. Even in institutional deliveries, delayed maternity admissions (often in prolonged or obstructed labor due to the lack of effective transport systems) may mean that the time window for initiation of therapeutic hypothermia is already lost by the time of delivery.

On the other hand, with rapidly improving standards of healthcare in urban settings and referral facilities available in transitional economies such as China, India, and Brazil, a large number of deliveries occur in hospitals, or infants may be admitted within the window period for initiation of rescue hypothermic neuroprotection. In fact, in the last two to three decades, the 
number of neonatal intensive care units and special care neonatal units in transitional countries such as India and China has increased exponentially $[12,13]$. Approximately $75 \%$ of deliveries in India now occur in hospitals, though this varies from 50\% in North India to $95 \%$ in South India $[14,15]$. Although accurate morbidity statistics are lacking, at least one million babies are likely to suffer from encephalopathy in India every year, and up to half of these deliveries may occur in a hospital setting. If therapeutic hypothermia were available to even a small proportion of these hospitals, it may provide substantial health benefits.

\subsection{The use of therapeutic hypothermia outside optimal tertiary neonatal intensive care}

Interventions that are safe and highly effective within well-resourced tertiary intensive care units may not be so elsewhere. For example, the use of fluid bolus in septic shock is a routine clinical practice in well-resourced pediatric intensive care units with facilities for cardiorespiratory support and invasive monitoring of vital signs. However, in Sub-Saharan African hospitals without these facilities, the intervention was associated with increased mortality [16].

It is unclear how much intensive care support is required for the safe administration of therapeutic hypothermia. The earliest therapeutic hypothermia trials in high-income countries were conducted only in tertiary neonatal units with facilities for optimal cardiorespiratory support and neurological monitoring, and the vast majority of the babies were kept ventilated and sedated during therapy. As experience with therapeutic hypothermia has increased, it is now offered by many secondary care units in the UK; and indeed many babies (especially with moderate encephalopathy) are not ventilated, or are extubated soon after initial resuscitation while undergoing therapeutic hypothermia. Most infants undergoing therapeutic hypothermia receive sedation in the UK; however, such routine sedation was not used in the NICHD cooling trial [17] and is not part of routine clinical practice in the NICHD Neonatal Research Network (NRN) in the USA (personal communication: Prof. Seetha Shankaran). In addition, studies have now shown that therapeutic hypothermia improves respiratory, renal, and other metabolic parameters in encephalopathic babies $[18,19]$. Thus, there is no reason to believe that therapeutic hypothermia may be ineffective without ventilatory support (although caution will need to be exercised in babies with persistent pulmonary hypertension from meconium aspiration). On the contrary, it is possible that the infants with moderate encephalopathy not requiring ventilatory support might be those who benefit most from therapeutic hypothermia in LMIC, and may achieve normal outcomes. Finally, many encephalopathic babies have a low core body temperature during first $24 \mathrm{~h}$ after birth [20], although this might be more apparent in babies born in low-resource settings, where radiant warmers are not routinely used [21]. It is unclear whether this hypothermia would have any neuroprotective effect, or whether rewarming these babies would cause more harm or benefit [22]. 
Unfortunately, the use of therapeutic hypothermia in low-resource settings has received bad publicity after a pilot study in Sub-Saharan Africa showed five-fold higher mortality in cooled babies, albeit statistically non-significant (RR: 5.0; 95\% CI: 0.7-37) [21]. It remains unclear whether this was related to a lack of basic neonatal care facilities and medical/nursing expertise in this setting (for example adequate neonatal resuscitation and routine monitoring of basic physiology); inadequate cooling devices (water bottles); lack of sedation [23]; or due to the recruitment of more severely encephalopathic infants in the cooled arm. Nevertheless, therapeutic hypothermia has now disappeared from the priority list of global health researchers, with infection being highlighted as a potential cause for the increased mortality with therapeutic hypothermia [24], despite little evidence to support this. This situation is not dissimilar to the 1960s, when therapeutic hypothermia received negative publicity after a clinical trial showed an increased mortality of hypothermic preterm infants [25]. Therapeutic hypothermia then disappeared from the clinic for several decades before it was rigorously re-evaluated in high-income countries. A repeat of this history would be unfortunate, and may deprive babies in LMIC from the benefits of one of the most important and simple interventions in medicine.

\section{Concerns about perinatal infection}

Fetal inflammation and infection has been shown to increase brain vulnerability to hypoxia-ischemia via stimulation of immune and inflammatory responses, chemotaxis, toll-like receptors and cell death [26]. Emerging experimental data also suggest that hypothermia may not be neuroprotective after bacterial lipopolysaccharide-sensitized NE brain injury as compared to hypothermia without bacterial lipopolysaccharide [27]. Hence, therapeutic hypothermia in the presence of infection might even be deleterious as hypothermia may impair innate immune function, including neutrophil migration and function [28].

In a prospective study, Tann et al. [29] reported that the prevalence of neonatal bacteremia with a pathogenic organism among encephalopathic infants was $3.5 \%$ by blood culture alone, $6.9 \%$ by polymerase chain reaction (PCR) alone, and $8.9 \%$ by blood culture and PCR in combination. A similar incidence of coexistent bloodstream-positive infection and encephalopathy has been reported from India [30,31]. This is similar to that reported from high-income countries: $8.1 \%$ in the ICE trial [32], 6\% in NICHD [17], 17\% in the TOBY registry [33]. Therefore, coexistent perinatal infection is unlikely to have a role in influencing the treatment efficacy of therapeutic hypothermia for the vast majority of babies in LMIC.

\section{Concerns about established brain injury}

Cerebral magnetic resonance imaging (MRI) in high-income countries has shown that cerebral injury is acute and acquired perinatally, and is not established (antenatal injury) at the time 
of birth; hence it is amenable to therapeutic hypothermia [34]. Emerging evidence from LMIC suggests similar perinatal origins of brain injury. Tann et al. [35] reported that $80 \%$ of the early cranial ultrasound (USG) scans were normal in encephalopathic babies admitted to a Sub-Saharan African neonatal unit, and none of them had evidence of established brain injury. Although the utility of USG in term encephalopathic infants is limited [36], these findings are similar to the cerebral MRI reported by Lally et al. from a South Indian cohort $(n=54)$, in which none of the encephalopathic babies had evidence of established brain injury. However, unlike in high-income countries, the predominant injury was in the white matter, rather than in the deep gray matter nuclei. Reduced white matter fractional anisotropy was associated with adverse neurological outcomes at three-and-a-half years in this population (Figs 1,2). Thus, it is possible that the brain injury in LMIC encephalopathic babies may be ameliorated with therapeutic hypothermia.

\section{Health and economic impact of cooling}

Neurodisability related to NE is a lifelong condition placing substantial burden on individuals, their families, and wider society. Therapeutic hypothermia reduces long-term neurodisability after neonatal encephalopathy; hence, in high-income countries where this intervention is applied widely, it has important health and economic benefits. Azzopardi et al. showed that, with respect to the current lifetime costs for each child with cerebral palsy (about $£ 750,000$ ) [37] and the economic benefit of additional healthy lives (about $£ 800,000$ ) [38], the total benefit to the UK economy as a result of the implementation of therapeutic hypothermia is likely to be in excess of $£ 125$ milion so far, despite the high cost of cooling devices [9].

As the burden of NE is far higher in LMIC $[2,39]$, the health and economic benefit of therapeutic hypothermia may be substantially higher in these settings, if it can be delivered via the existing infrastructure using inexpensive cooling devices. Nevertheless, health economic evaluation in LMIC is challenging due to the lack of accurate data on cost estimates of neonatal encephalopathy and neurodisability, and because expenses are borne by individual families rather than by healthcare systems.

\section{Ethical aspects of cooling therapy}

To some, controlled trials of hypothermia in LMIC are already entering an ethical gray area. For example, the World Medical Association Declaration of Helsinki specifically indicates that comparisons with no treatment or placebo are only appropriate where there is no current proven intervention [40]. Others argue that it can be neither acceptable nor safe to extrapolate the evidence from high-income country therapeutic hypothermia trials performed under optimal intensive care settings to LMIC, where the population comorbidities and neonatal care are very different. Furthermore, maintaining normothermia is one of the founding pillars of newborn survival in LMIC 
due to the strong epidemiological association of hypothermia and neonatal mortality. Very strong evidence is required before this conventional wisdom is challenged in LMIC.

Although there have been past concerns that therapeutic hypothermia might convert death into disability for those babies with severe encephalopathy (an extremely undesirable situation, particularly in LMIC), the data from therapeutic hypothermia trials have not supported this hypothesis [6]. Therapeutic hypothermia not only reduces mortality, but also decreases neurodisability in survivors [6]. Furthermore, most of the babies recruited to LMIC therapeutic hypothermia trials appear to have moderate rather than severe encephalopathy (Table 1); these babies are more likely to benefit from therapeutic hypothermia [6].

Another argument might be that resources should be invested to improve poor antenatal care, thus preventing neonatal encephalopathy rather than treating it. On the other hand, given the large number of infants affected by neonatal encephalopathy, it is unreasonable not to explore therapeutic hypothermia for these babies - a simple and potential highly effective neuroprotectant therapy.

\section{Cooling equipment}

The equipment used to provide therapeutic hypothermia in developed countries is prohibitively expensive and unsuitable for wide use in LMIC. Instead, several low-technology devices have been used for cooling therapy in LMIC (Fig. 3). A brief summary is given below.

\subsection{Passive cooling}

Several studies have reported use of passive cooling (i.e keeping baby naked without the use of radiant warmer) for induction of cooling and during transport. Although this facilitates early induction of cooling, significant overcooling $\left(<32^{\circ} \mathrm{C}\right)$ has been reported in up to one-third of the infants $[41,42]$. It is unlikely that the target temperatures can be maintained by passive cooling alone.

\subsection{Water and ice}

Effective whole-body cooling has been reported using water bottles and gloves [43] filled with water. Selective head cooling using ice may result in marked temperature fluctuations, and effective whole-body cooling has been achieved using ice and frozen gel packs in some studies. Although therapeutic hypothermia with ice showed improved outcomes in high-income tertiary neonatal units with 1:1 nursing care [44], the vast majority of neonatal units in LMIC do not have such nursing and monitoring resources. In our experience, cooling using ice may result in wide temperature fluctuations without close nursing monitoring.

\subsubsection{Laminar flow device}


Perez et al. [45] have reported effective whole-body cooling in 23 babies with encephalopathy admitted to a Brazilian neonatal unit using a laminar flow device (Neonatflow). Hypothermia could be induced within 90 min in all babies.

\subsubsection{Cooling using fans}

A servo-controlled cooling fan developed by Horn et al. [46] appears to be an effective lowcost cooling device, and a feasibility study on 10 babies using this device has been reported. Five of these babies had shivering at higher fan speeds.

\subsubsection{Clay pot cooling}

Kim et al. have developed an interesting cooling device using two clay pots, sand, and a urea-based powder. When water is added to the mixture of powder and sand, the powder dissolves and leaks through the outer clay pot. Hypothermia is achieved by evaporative cooling, and has been shown to be effective in a newborn piglet model [47].

\subsection{Phase change materials}

Phase change materials (PCM) that can be used for medicine are normally made of salt hydride, fatty acid, and esters of paraffin and melt (or sets) at a set temperature [48]. One useful composition is based on Glauber salt, modified by varying the amount of sodium chloride and additional components to achieve desired phase change properties. The ability of PCM to maintain a steady temperature by storing or releasing a large amount of thermal energy provides a natural feedback loop. Outside the phase-change temperature range, these materials act like any other substance. Effective hypothermia was induced and maintained using phase-changing material with a set temperature of $32^{\circ} \mathrm{C}$ in encephalopathic babies in south India (approximate cost US\$200); however, this was effective only when the ambient temperature was $<28^{\circ} \mathrm{C}$ and additional nursing input was required to maintain the target temperature, limiting wider use in tropical countries as a cooling device [49].

\subsection{Affordable servo-controlled cooling devices}

The emerging data suggest that therapeutic hypothermia is effective only within a narrow range of $33-34^{\circ} \mathrm{C}$, and elective cooling to $32^{\circ} \mathrm{C}$ for three or five days may worsen outcomes [50], hence the importance of tight temperature control during cooling therapy should not be underestimated. It is unlikely that manual cooling devices are able to achieve such tight temperature control without additional nursing resources that may offset the cost benefit of an inexpensive cooling method. Hence, another alternative is simplified and affordable versions of existing highincome country servo-controlled cooling devices modified for LMIC conditions. TecothermHELIX is one such servo-controlled cooling device developed for exclusive use in LMIC (estimated to cost US\$1000). It works by single-switch operation to achieve a set temperature of 
$33.5^{\circ} \mathrm{C}$, re-circulating water through a re-usable cooling mattress. It can also work on battery and have no ongoing cost. Feasibility studies using this device in south India show effective cooling times [51] similar to the expensive high-income country cooling devices, with minimal additional nursing input and ongoing costs [52].

\section{Low- and middle-income country cooling trials}

Although a number of pilot therapeutic hypothermia studies have been reported from LMIC, none of these studies was adequately powered to examine clinically important outcomes (Table 1). The largest of these was from China, which unfortunately was of poor quality. Zhou et al. [53] reported 18-month outcome data on 256 babies randomized to selective head cooling or usual care. Infants at risk of sepsis were excluded, and there were post-randomization exclusions. A homemade head-cooling device was used, but details were unclear as there was no documented trade name on this equipment. It is likely that the investigators cooled the body via the head, and that this may be whole-body cooling rather than selective head cooling, which requires heating of the body to obtain a head-to-body gradient. Twenty four percent were lost to follow-up. Although the authors claimed that selective cooling improved outcomes, the validity of this claim is questionable [53].

More worrying is the very low mortality rate $(<5 \%)$ reported in some therapeutic hypothermia studies from India on infants who were reported to have moderate or severe encephalopathy $[32,54]$. Although the exact reasons are unclear, this is likely to be due to lack of a standardized neurological examination to define encephalopathy, thus potentially including babies without encephalopathy based on low Apgar scores alone. Such a practice may expose babies with a very low risk of adverse outcome to the adverse effects of unnecessary therapeutic hypothermia.

A recent meta-analysis of therapeutic hypothermia in LMIC has shown that the pooled data from seven randomised controlled trials [21,31,49,53,55-57], including a total of 567 infants, showed a reduction in neonatal mortality, although this was not statistically significant (RR: 0.74; 95\% CI: 0.44-1.25) [58]. This observation has been confirmed in another systematic review by Galvao et al. [59], which showed that studies in lower income economies did not have statistical power to detect significant effects of hypothermia after stratification by country income. Interestingly, this meta-regression failed to show that countries' gross domestic product per capita (an indirect measure of healthcare resources) affects the efficacy of hypothermia [59].

Unfortunately, the poor quality of the trials in both of these systematic reviews [21,31,49,53,55-57], including the lack of comprehensive follow-up of trial subjects, currently provides no evidence for either safety or efficacy in the use of hypothermia as a neuroprotectant for neonatal encephalopathy in LMIC. 
Another challenge is assessing long-term neurodevelopmental outcome after encephalopathy due to a lack of robust standardized tools for the local population, particularly with regard to language assessments and high attrition rates. There have been concerns about the artificial lowering of scores yielded by tools typically applied in developed countries (e.g. Bayley III and Griffiths) when used in LMIC. However, in our experience this has not been an issue, and the Bayley III scores on healthy south Indian children were similar to the UK scores. Unfortunately, these tools are prohibitively expensive for routine use in LMIC at present.

\section{The way forward}

Although there are differences in population comorbidities and in the underlying brain injury after neonatal encephalopathy, the therapeutic benefits of hypothermia when used within the existing healthcare infrastructure in LMIC (i.e. without optimal tertiary intensive care and respiratory support) cannot be excluded. Indeed, the health and economic benefit of therapeutic hypothermia may be far higher in LMIC than in high-income countries, considering the disease burden. However, adequate powered clinical trials are required to ensure that cooling is indeed safe and effective in LMIC neonatal units, before routine clinical use can be recommended. One such large multi-country cooling trial in LMIC (HELIX trial: Hypothermia for Encephalopathy in Low and Middle-Income Countries) is currently in progress

(http://clinicaltrials.gov/show/NCT01760629).

Without such trials, therapeutic hypothermia will creep into use in LMIC, but wider adoption will be constantly sabotaged by residual safety concerns; thus, one of the greatest discoveries in neonatal medicine may be lost in translation. Policy changes and incorporation of therapeutic hypothermia into the World Health Organization guidelines are unlikely to occur without robust evidence of its safety and efficacy. Meanwhile, it would be prudent for enthusiastic clinicians offering therapeutic hypothermia in LMIC to adhere to the therapeutic hypothermia guidelines and inclusion criteria established in high-income countries.

\section{Practice points}

- Although cooling is a highly effective therapy for neonatal encephalopathy in high-income countries, its safety and efficacy in LMIC is unclear.

- Clinicians offering cooling therapy in LMIC should adhere to the established protocols from high-income countries, and offer this only to infants with moderate or severe encephalopathy as assessed using a structured neurological examination.

- Inexpensive manual cooling methods such as ice and gel packs require close nursing monitoring to maintain core temperature within the target range, limiting wider use in LMIC. 


\section{Research directions}

- Investigations of the nature and origins of brain injury after neonatal encephalopathy, its interaction with perinatal infections, the implications for long-term neurological outcomes, and its impact on the health and economic burden in LMIC.

- Clinical trials to establish the safety and efficacy of cooling therapy in LMIC.

- Development of affordable servo-controlled cooling devices suitable for use in LMIC.

\section{Funding sources}

Bill and Melinda Gates foundation (HELIX trial); Biomedical Research Centre, Imperial 


\section{Table 1}

Published studies on cooling after neonatal encephalopathy in low- and middle-income countries

\begin{tabular}{|c|c|c|c|c|c|c|c|}
\hline Country & Study & $N$ & $\begin{array}{l}\text { Type of } \\
\text { study }\end{array}$ & Cooling device & $\mathrm{WBC} / \mathrm{SHC}$ & $\begin{array}{l}\text { Grade of HIE } \\
\text { Mild/moderate/severe }\end{array}$ & Neonatal mortality (\%) \\
\hline \multirow[t]{6}{*}{ Africa } & Horn et al. [60] & $4 \mathrm{H}$ & Case series & $\begin{array}{l}\text { Insulated ice } \\
\text { caps }\end{array}$ & SHC & Not mentioned & Not reported \\
\hline & $\begin{array}{l}\text { Robertson et al. } \\
{[21]}\end{array}$ & $\begin{array}{l}36 \\
21 \mathrm{H}, 15 \mathrm{~S}\end{array}$ & $\mathrm{RCT}$ & Water bottles & WBC & $9 / 20 / 7$ & $33.3 \mathrm{H}, 6.6 \mathrm{~S}$ \\
\hline & Horn et al. [46] & $10 \mathrm{H}$ & Case series & $\begin{array}{l}\text { Servo-controlled } \\
\text { fans }\end{array}$ & $\mathrm{SHC}$ & $0 / 6 / 4$ & $10 \mathrm{H}$ \\
\hline & Horn et al. [61] & $5 \mathrm{H}$ & Case series & Frozen gel packs & SHC & $1 / 2 / 2$ & Not reported \\
\hline & Horn et al. [62] & $14 \mathrm{H}$ & Case series & Frozen gel packs & $\mathrm{SHC}$ & $\begin{array}{l}14 \text { moderate to } \\
\text { severe }\end{array}$ & $21.4 \mathrm{H}$ \\
\hline & Biselele et al. [63] & $50 \mathrm{H}$ & $\begin{array}{l}\text { Prospective } \\
\text { cohort study }\end{array}$ & Not cooled & NA & $16 / 20 / 8$ & $28 \mathrm{H}$ \\
\hline \multirow[t]{4}{*}{ India } & Bhatt et al. [57] & $\begin{array}{l}35 \\
20 \mathrm{H}, 15 \mathrm{~S}\end{array}$ & $\mathrm{RCT}$ & Not described & WBC & $\begin{array}{l}\text { Severe birth asphyxia } \\
(5 \mathrm{~m} \text { Apgar }<3)\end{array}$ & $15 \mathrm{H}, 33.3 \mathrm{~S}$ \\
\hline & Rajhans et al. [64] & $5 \mathrm{H}$ & Case series & Blanketrol II & WBC & $\begin{array}{l}\text { All moderate to } \\
\text { severe }\end{array}$ & Not reported \\
\hline & Thomas et al. [54] & $20 \mathrm{H}$ & Case series & Frozen gel packs & WBC & $0 / 16 / 4$ & $5 \mathrm{H}$ \\
\hline & $\begin{array}{l}\text { Bharadwaj et al. } \\
\text { [31] }\end{array}$ & $\begin{array}{l}124 \\
62 \mathrm{H}, 62 \mathrm{~S}\end{array}$ & $\mathrm{RCT}$ & Frozen gel packs & WBC & $0 / 109 / 15$ & $4.8 \mathrm{H}, 9.7 \mathrm{~S}$ \\
\hline
\end{tabular}




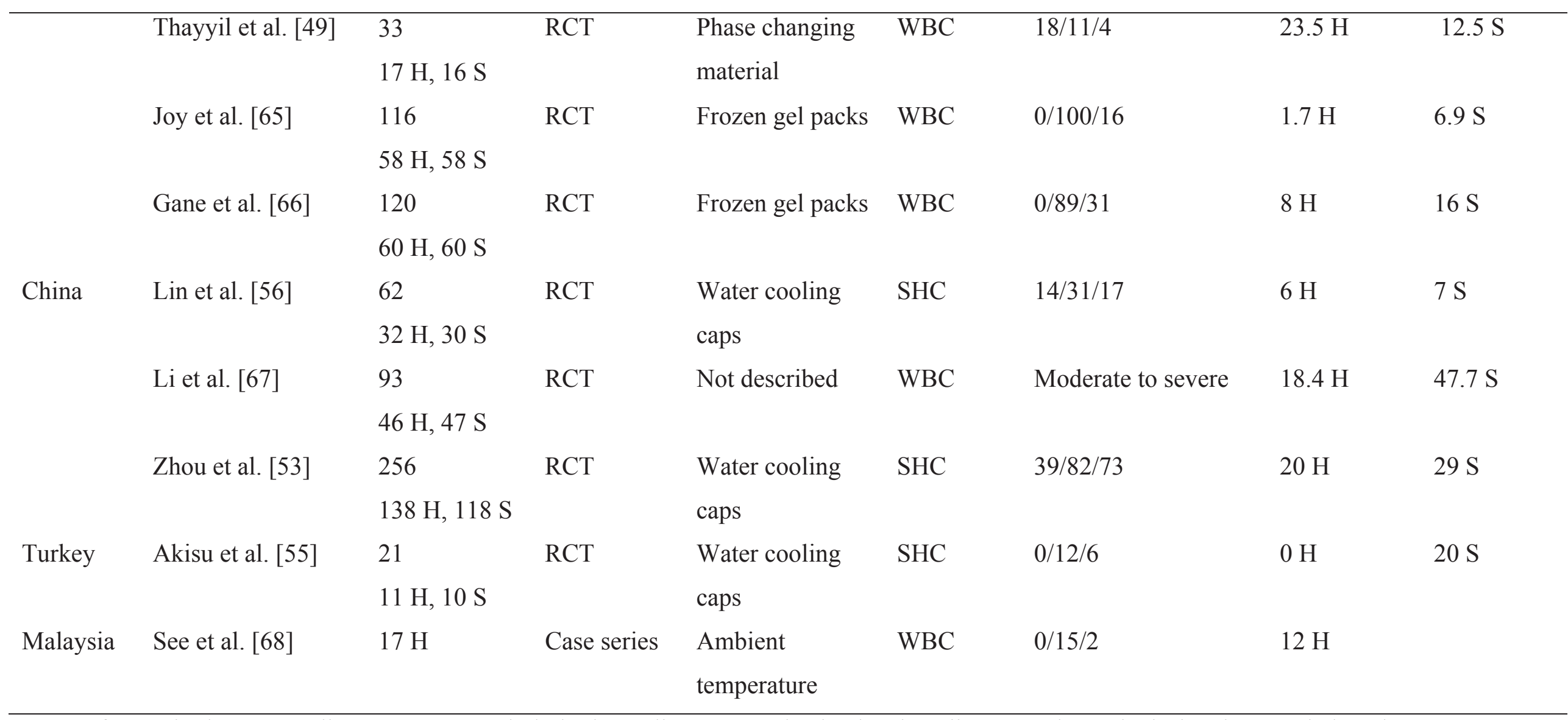

$n$, no. of cases in the non-cooling arm; WBC, whole-body cooling; SHC selective head cooling; HIE, hypoxic-ischemic encephalopathy; RCT, randomized controlled trial; $\mathrm{H}$, hypothermic arm; $\mathrm{S}$, standard care arm. 


\section{References}

[1] Kurinczuk JJ, White-Koning M, Badawi N. Epidemiology of neonatal encephalopathy and hypoxic-ischaemic encephalopathy. Early Hum Dev 2010;86:329-38.

[2] Lawn J, Shibuya K, Stein C. No cry at birth: global estimates of intrapartum stillbirths and intrapartum-related neonatal deaths. Bull WHO 2005;83:409-17.

[3] Ellis M, Manandhar N, Shrestha PS, Shrestha L, Manandhar DS, Costello AM. Outcome at 1 year of neonatal encephalopathy in Kathmandu, Nepal. Dev Med Child Neurol 1999;41:689-95.

[4] Lawn JE, Cousens S, Zupan J. 4 million neonatal deaths: when? Where? Why? Lancet 2005;365:891-900.

[5] Edwards AD. The discovery of hypothermic neural rescue therapy for perinatal hypoxic-ischemic encephalopathy. Semin Pediatr Neurol 2009;16:200-6.

[6] Jacobs SE, Berg M, Hunt R, Tarnow-Mordi WO, Inder TE, Davis PG. Cooling for newborns with hypoxic ischaemic encephalopathy. Cochrane Database Syst Rev 2013;(1):CD003311.

[7] Shankaran S, Pappas A, McDonald SA, et al. Childhood outcomes after hypothermia for neonatal encephalopathy. N Engl J Med 2012;366:2085-92.

[8] Azzopardi D, Strohm B, Marlow N, et al. Effects of hypothermia for perinatal asphyxia on childhood outcomes. N Engl J Med 2014;371:140-9.

[9] Azzopardi D, Strohm B, Linsell L, et al. Implementation and conduct of therapeutic hypothermia for perinatal asphyxial encephalopathy in the UK - analysis of national data. PLoS One 2012;7:e38504.

[10] Paul VK, Singh M. Regionalized perinatal care in developing countries. Semin Neonatol 2004;9:117-24.

[11] Barros AJ, Ronsmans C, Axelson H, et al. Equity in maternal, newborn, and child health interventions in Countdown to 2015: a retrospective review of survey data from 54 countries. Lancet 2012;379:1225-33.

[12] Coordination Group for National Survey of Development in Pediatric and Neonatal Intensive Care Units. Development of pediatric and neonatal intensive care units: results of a national survey (2000-2009). Zhonghua Er Ke Za Zhi 2011;49:669-74.

[13] Sundaram V, Chirla D, Panigrahy N, Kumar P. Current status of NICUs in India: a nationwide survey and the way forward. Indian J Pediatr 2014 (Jun 15) [Epub ahead of print].

[14] National Neonatology Forum in partnership with the Ministry of Health and Family Welfare Government of India, the World Health Organization (South East Asia 
Region), UNICEF India, the World Bank, and Saving Newborn Lives, Save the Children/US. State of India's Newborns. New Delhi/Washington DC; 2004.

[15] Nath A. India's progress toward achieving the millennium development goals. Indian J Community Med 2011;36:85-92.

[16] Maitland K, Kiguli S, Opoka RO, et al. Mortality after fluid bolus in African children with severe infection. N Engl J Med 2011;364:2483-95.

[17] Shankaran S, Laptook AR, Ehrenkranz RA, et al. Whole-body hypothermia for neonates with hypoxic-ischemic encephalopathy. N Engl J Med 2005;353:1574-84.

[18] Dassios T, Austin T. Respiratory function parameters in ventilated newborn infants undergoing whole body hypothermia. Acta Paediatr 2014;103:157-61.

[19] Sarkar S, Barks JD. Barks, systemic complications and hypothermia. Semin Fetal Neonatal Med 2010;15:270-5.

[20] Burnard ED, Cross KW. Rectal temperature in the newborn after birth asphyxia. BMJ 1958;2:1197-9.

[21] Robertson NJ, Nakakeeto M, Hagmann C, et al. Therapeutic hypothermia for birth asphyxia in low-resource settings: a pilot randomised controlled trial. Lancet 2008;372:801-3.

[22] Paul VK. The current state of newborn health in low income countries and the way forward. Semin Fetal Neonatal Med 2006;11:7-14.

[23] Thoresen M, Satas S, Løberg EM, et al. Twenty-four hours of mild hypothermia in unsedated newborn pigs starting after a severe global hypoxic-ischemic insult is not neuroprotective. Pediatr Res 2001;50:405-11.

[24] Lawn JE, Bahl R, Bergstrom S, et al. Setting research priorities to reduce almost one million deaths from birth asphyxia by 2015. PLoS Med 2011;8:e1000389.

[25] Silverman WA, Fertig JW, Berger AP. The influence of the thermal environment upon the survival of newly born premature infants. Pediatrics 1958;22:876-86.

[26] Stridh L, Mottahedin A, Johansson ME, et al. Toll-like receptor-3 activation increases the vulnerability of the neonatal brain to hypoxia-ischemia. J Neurosci 2013;33:12041-51.

[27] Osredkar D, Thoresen M, Maes E, Flatebø T, Elstad M, Sabir H. Hypothermia is not neuroprotective after infection-sensitized neonatal hypoxic-ischemic brain injury. Resuscitation 2014;85:567-72.

[28] Biggar WD, Barker C, Bohn D, Kent G. Partial recovery of neutrophil functions during prolonged hypothermia in pigs. J Appl Physiol 1986;60:1186-9. 
[29] Tann CJ, Nkurunziza P, Nakakeeto M, et al. Prevalence of bloodstream pathogens is higher in neonatal encephalopathy cases vs. controls using a novel panel of real-time PCR assays. PLoS One 2014;9:e97259.

[30] Lally PJ, Price DL, Pauliah SS, et al. Neonatal encephalopathic cerebral injury in South India assessed by perinatal magnetic resonance biomarkers and early childhood neurodevelopmental outcome. PLoS One 2014;9:e87874.

[31] Bharadwaj SK, Bhat BV. Therapeutic hypothermia using gel packs for term neonates with hypoxic ischaemic encephalopathy in resource-limited settings: a randomized controlled trial. J Trop Pediatr 2012;58:382-8.

[32] Jacobs SE, Morley CJ, Inder TE, et al. Whole-body hypothermia for term and near-term newborns with hypoxic-ischemic encephalopathy: a randomized controlled trial. Archs Pediatr Adolesc Med 2011;165:692-700.

[33] Azzopardi DV, Strohm B, Edwards AD, et al. Moderate hypothermia to treat perinatal asphyxial encephalopathy. N Engl J Med 2009;361:1349-58.

[34] Cowan F, Rutherford M, Groenendaal F, et al. Origin and timing of brain lesions in term infants with neonatal encephalopathy. Lancet 2003;361:736-42.

[35] Tann CJ, Nakakeeto M, Hagmann C, et al. Cranial ultrasound findings suggest that the injury pathway may begin many hours before delivery in encephalopathic infants in Uganda. Archs Dis Childh Fetal Neonatal Ed 2014;99 Suppl 1:A14-15.

[36] Boo NY, Chandran V, Zulfiqar MA, et al. Early cranial ultrasound changes as predictors of outcome during first year of life in term infants with perinatal asphyxia. J Paediatr Child Health 2000;36:363-9.

[37] Kruse M, Michelsen SI, Flachs EM, et al. Lifetime costs of cerebral palsy. Dev Med Child Neurol 2009;51:622-8.

[38] Grosse SD, Krueger KV, Mvundura M. Economic productivity by age and sex: 2007 estimates for the United States. Med Care 2009;47(7 Suppl 1):S94-103.

[39] Thayyil S, Bhutta ZA, Ramji S, Costello AM, Robertson NJ. Global application of therapeutic hypothermia to treat perinatal asphyxial encephalopathy. Int Health 2010;2:79-81.

[40] World Medical Association. Declaration of Helsinki (October 2008). http://www.wma.net/e/policy/b3.htm (accessed 16 August 2010).

[41] Fairchild K, Sokora D, Scott J, Zanelli S. Therapeutic hypothermia on neonatal transport: 4-year experience in a single NICU. J Perinatol 2010;30:324-9. 
[42] Akula VP, Gould JB, Davis AS, et al. Therapeutic hypothermia during neonatal transport: data from the California Perinatal Quality Care Collaborative (CPQCC) and California Perinatal Transport System (CPeTS) for 2010. J Perinatol 2013;33:194-7.

[43] Hoque N, Chakkarapani E, Liu X, Thoresen MA. comparison of cooling methods used in therapeutic hypothermia for perinatal asphyxia. Pediatrics 2010;126:e124-30.

[44] Jacobs SE, Morley CJ, Inder TE, et al. Whole-body hypothermia for term and near-term newborns with hypoxic-ischemic encephalopathy: a randomized controlled trial. Archs Pediatr Adolesc Med 2011;165:692-700.

[45] Perez JMR, Golombek SG, Alpan G, Fajardo C A, Sola A. Comparison of hypothermia for hypoxic-ischemic encephalopathy with a laminar flow unit (neonatflow) vs. NICHD Trial. Pediatric Academic Society Meeting, Vancouver, Canada, 2014.

[46] Horn A, Thompson C, Woods D, et al. Induced hypothermia for infants with hypoxic-ischemic encephalopathy using a servo-controlled fan: an exploratory pilot study. Pediatrics 2009;123:e1090-8.

[47] Kim JJ, Buchbinder N, Ammanuel S, et al. Cost-effective therapeutic hypothermia treatment device for hypoxic ischemic encephalopathy. Med Devices (Auckl) 2013;6:1-10.

[48] Olson L. On neonatal asphyxia: clinical and animal studies including development of a simple, safe method for therapeutic hypothermia with global applicability. $\mathrm{PhD}$ thesis, Karolinska Institutet, Stockholm; 2011.

[49] Thayyil S, Shankaran S, Wade A, et al. Whole-body cooling in neonatal encephalopathy using phase changing material. Archs Dis Childh Fetal Neonatal Ed 2013;98:F280-1.

[50] Shankaran S, Laptook A, Pappas A, et al. Randomized controlled trial (RCT) of optimizing cooling strategies for neonatal hypoxic-ischemic encephalopathy (HIE). Pediatric Academic Society Meeting, Vancouver, 2014.

[51] Robertson NJ, Kendall GS, Thayyil S. Techniques for therapeutic hypothermia during transport and in hospital for perinatal asphyxial encephalopathy. Semin Fetal Neonatal Med 2010;15:276-86.

[52] Pauliah S, Narayanan E, Kumutha K, et al. Hypothermia for Encephalopathy in Low and Middle-Income Countries (HELIX): a feasibility study. Archs Dis Childh Fetal Neonatal Ed 2014;99:A74.

[53] Zhou WH, Cheng GQ, Shao XM, et al. Selective head cooling with mild systemic hypothermia after neonatal hypoxic-ischemic encephalopathy: a multicenter randomized controlled trial in China. J Pediatr 2010;157:367-72. 
[54] Thomas N, George KC, Sridhar S, et al. Whole body cooling in newborn infants with perinatal asphyxial encephalopathy in a low resource setting: a feasibility trial. Indian Pediatr 2011;48:445-51.

[55] Akisu M, Huseyinov A, Yalaz M, Cetin H, Kultursay N. Selective head cooling with hypothermia suppresses the generation of platelet-activating factor in cerebrospinal fluid of newborn infants with perinatal asphyxia. Prostagl Leukot Essent Fatty Acids 2003;69:45-50.

[56] Lin ZL, Yu HM, Lin J, Chen SQ, Liang ZQ, Zhang ZY. Mild hypothermia via selective head cooling as neuroprotective therapy in term neonates with perinatal asphyxia: an experience from a single neonatal intensive care unit. J Perinatol 2006;26:180-4.

[57] Bhat MA. Re: Therapeutic hypothermia following perinatal asphyxia. Archs Dis Childh Fetal Neonatal Ed 2006;91:F465.

[58] Pauliah SS, Shankaran S, Wade A, Cady EB, Thayyil S. Therapeutic hypothermia for neonatal encephalopathy in low- and middle-income countries: a systematic review and meta-analysis. PLoS One 2013;8:e58834.

[59] Galvao TF, Silva MT, Marques MC, de Oliveira ND, Pereira MG. Hypothermia for perinatal brain hypoxia-ischemia in different resource settings: a systematic review. J Trop Pediatr 2013;59:453-9.

[60] Horn AR, Woods DL, Thompson C, Eis I, Kroon M. Selective cerebral hypothermia for post-hypoxic neuroprotection in neonates using a solid ice cap. S Afr Med J 2006;96:976-81.

[61] Horn AR, Harrison MC, Linley LL. Evaluating a simple method of neuroprotective hypothermia for newborn infants. J Trop Pediatr 2010;56:172-7.

[62] Horn AR, Joolay Y, Tooke L, Harrison MC. A servo-assisted gel-pack cooling method for newborn infants with hypoxic-ischemic encephalopathy. J Trop Pediatr 2012;58:236-8.

[63] Biselele T, Naulaers G, Bunga Muntu P, et al. A descriptive study of perinatal asphyxia at the University Hospital of Kinshasa (Democratic Republic of Congo). J Trop Pediatr 2013;59:274-9.

[64] Rajhans AP, Chouthai NS, Joshi RV. Whole body hypothermia (WBH) for newborns with moderate to severe hypoxic ischemic encephalopathy (HIE) in India. Boston: Pediatric Academic Society; 2012.

[65] Joy R, Pournami F, Bethou A, Bhat VB, Bobby Z. Effect of therapeutic hypothermia on oxidative stress and outcome in term neonates with perinatal asphyxia: a randomized controlled trial. J Trop Pediatr 2013;59:17-22. 
[66] Gane BD, Bhat V, Rao R, Nandhakumar S, Harichandrakumar KT, Adhisivam B. Effect of therapeutic hypothermia on DNA damage and neurodevelopmental outcome among term neonates with perinatal asphyxia: a randomized controlled trial. J Trop Pediatr 2014;60:134-40.

[67] Li T, Xu F, Cheng X, et al. Systemic hypothermia induced within 10 hours after birth improved neurological outcome in newborns with hypoxic-ischemic encephalopathy. Hosp Pract 2009;37:147-52.

[68] See KC, Jamal SJ, Chiam ML. Short term outcome of therapeutic hypothermia in term infants with moderate to severe hypoxic ischaemic encephalopathy; The Sungai Buloh Experience. Med J Malaysia 2012;67:265-8. 
Fig. 1. Axial T1 (lower left and centre)- and T2-weighted (top left and centre) magnetic resonance images acquired during the first week after birth in an infant with mild neonatal encephalopathy in south India. Extensive white matter injury (white arrows and blue arrowheads), and a small intraventricular bleed (red arrow) are seen. Hemiplegic cerebral palsy was noted on neurodevelopmental assessment at 3.5 years of age. Reproduced with permission from Lally et al. [30].

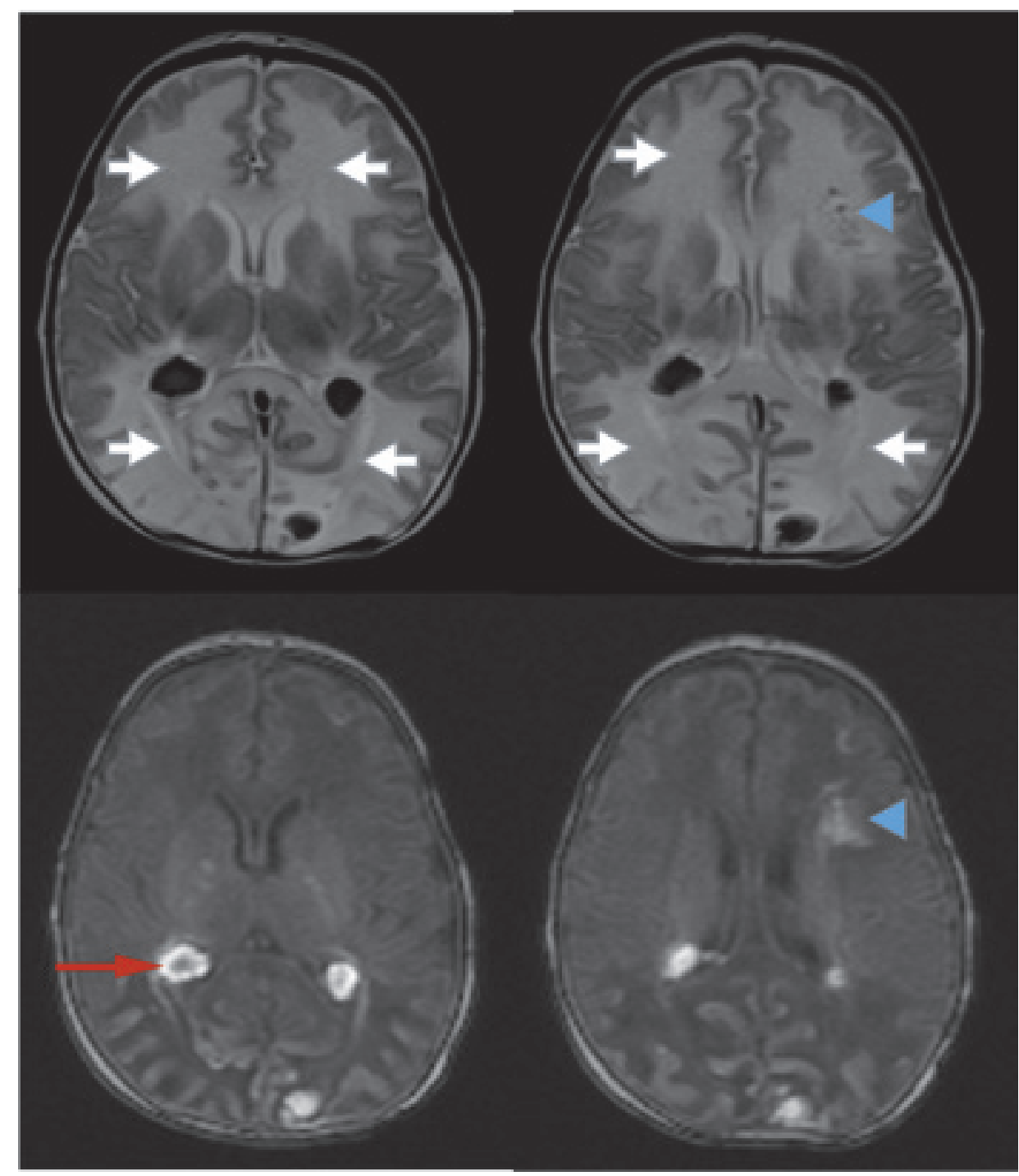


Fig. 2. Tract-based spatial statistics of whole-brain fractional anisotropy and long-term neurological outcomes after neonatal encephalopathy in south India. Axial (upper right) and coronal (lower right) sections of the cohort white matter skeleton (green) show significant reductions in fractional anisotropy (red-yellow) in children who had adverse neurological outcome $(n=4)$ after neonatal encephalopathy at 3.5 years of age, when compared with those who had favorable outcome $(N=21)$. Reproduced with permission from Lally et al. [30].

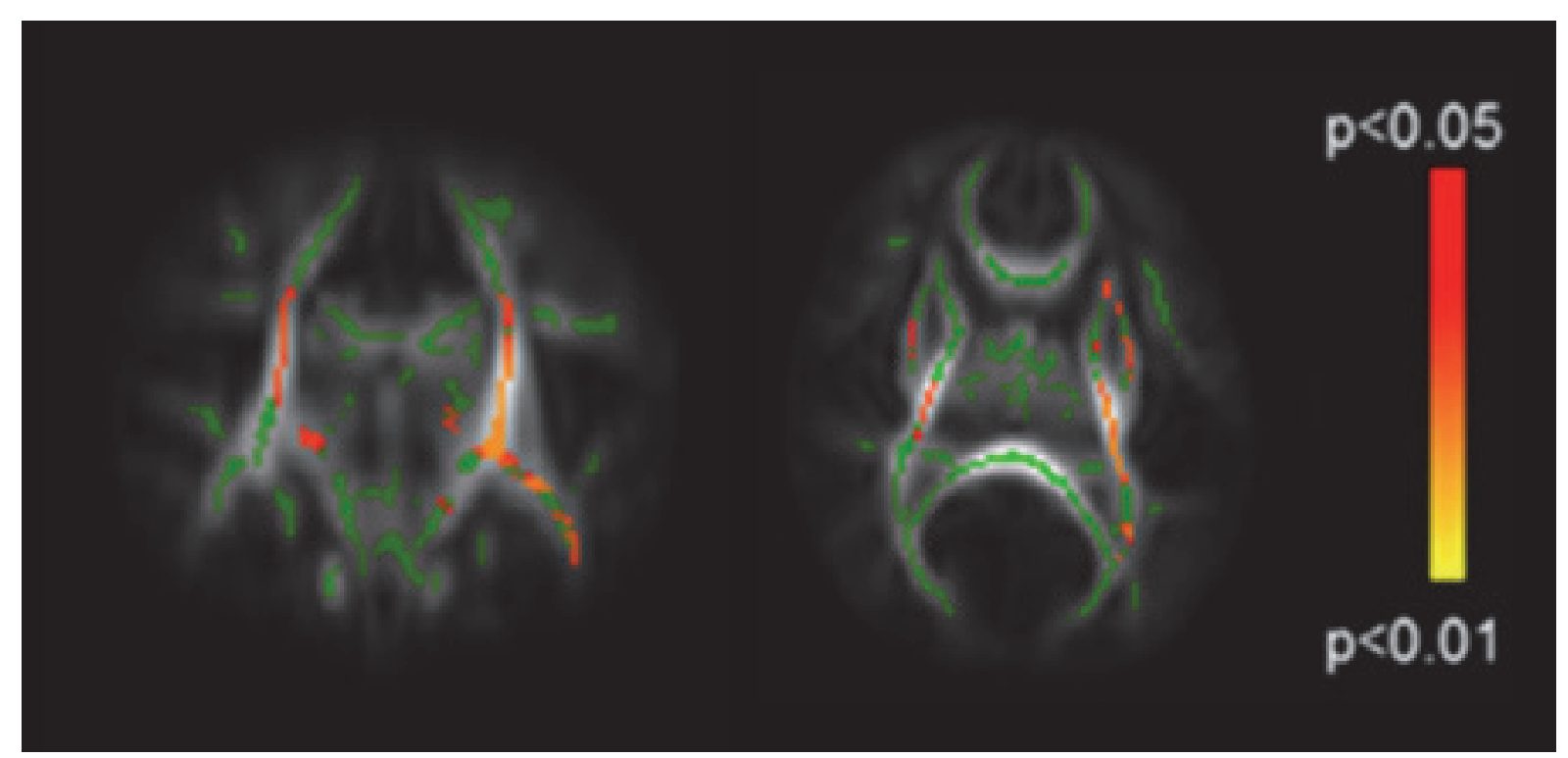


Fig. 3. Manual low-technology cooling devices. (i) Water bottle (bottle filled with tap water at $25^{\circ} \mathrm{C}$ and laid sideways under the infant in the cot). (ii) Phase change material mattress (PCM, melting point $32^{\circ} \mathrm{C}$ ). Principles of heat exchange (A); structure of the mattress (B), a baby receiving cooling by PCM at Government Medical College, Kozhikode, India (C); and passive re-warming (D). (iii) Cooling using frozen gel packs at Christian Medical College, Vellore, India (photograph courtesy of Niranjan Thomas). (iv) Clay pot cooling device.

Temperature changes are based on heat transfer, water diffusion, and air flow of the surrounding system. As water evaporates, heat is drawn from the inner clay pot.

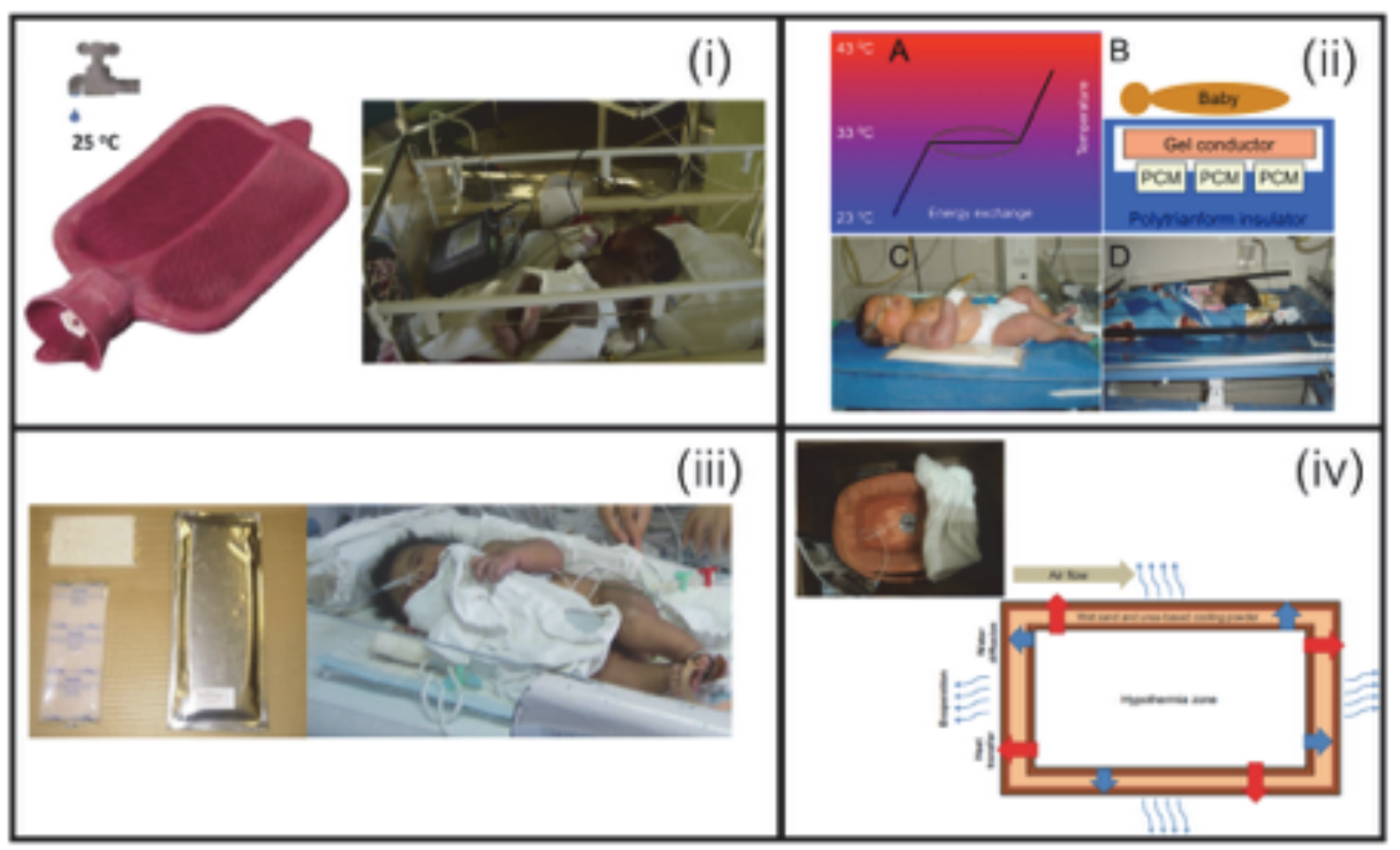

